\title{
PARA UNA HISTORIA DE LO IMPOSIBLE. LA HISTORIA DE LAS IDEAS FILOSÓFICAS EN WALTER BENJAMIN
}

\author{
FOR A HISTORY OF THE IMPOSSIBLE. THE HISTORY \\ OF PHILOSOPHICAL IDEAS IN WALTER BENJAMIN
}

\section{ALEJANDRO FIELBAUM S.*}

\section{RESUMEN}

El trabajo aborda el lugar de la historia de las ideas filosóficas en la concepción de Walter Benjamin de la historia de la cultura. Desde sus tempranas reflexiones sobre la inagotable trascendencia de la idea, Benjamin piensa en las formas en que distintos fenómenos históricos la inscriben siempre parcialmente. Tras la diferenciación moderna entre filosofía y literatura, el abordaje por el contenido de verdad de esta última es más interesante para Benjamin. Mientras la filosofía burguesa moderna se refugia en una limitada interioridad, en la literatura moderna Benjamin lee las tensiones de la modernidad, a partir de una consideración de la especificidad del texto literario cuyo contenido de verdad el filósofo podría desear desde cierta pregunta filosófica que no impone, a la literatura, sus esquemas conceptuales.

Palabras clave: Walter Benjamin, historia de las ideas, filosofía.

\section{ABSTRACT}

This article addresses the place of the history of philosophical ideas in Walter Benjamin's conception of cultural history. From his earlier reflections about the transcendence of the idea, he thinks of the different forms in which diverse historical phenomena always present it partially. After the modern separation of philosophy and literature,

* Sociólogo y licenciado en Filosofía por la Pontificia Universidad Católica de Chile. Magíster en Estudios Latinoamericanos, Universidad de Chile. Actualmente, cursa estudios de Doctorado en Estudios Latinoamericanos en la UNAM, y es coinvestigador del Proyecto Fondecyt "Filosofía y Literatura en América Latina (Fines del siglo XIX y primeras décadas del siglo XX)”. Correo electrónico: afielbaums@gmail.com 
the preoccupation for the content of truth of the latter will be more interesting for Benjamin. While modern and bourgeoisie philosophy houses itself in a limited interiority, modern literature exposes the tensions of modernity from a reading that considers the particularity of the literary text, whose content of truth the philosopher might desire from a certain philosophical question that does not impose, on literature, its conceptual schemas.

Keywords: Walter Benjamin, history of ideas, philosophy.

Recibido: 18.04.12. Aceptado: 21.10.16.

Nada vale la pena de ser hecho sino lo que el mundo juzga imposible.

(WILDE, 1920)

\section{WALTER BENJAMIN, DENTRO Y FUERA DE LA FILOSOFÍA}

WALTER

BENJAMIN

$(6)$
$\mathrm{B}$ UENA PARTE DE LAS LARGAS y necesarias discusiones sobre el pensamiento de Walter Benjamin, particularmente acerca de las tareas que su obra lega a un trabajo crítico del pensamiento contemporáneo, pasan por la ubicación de su trabajo en relación a la filosofía. Michael Lowy (2002, p. 10), por ejemplo, señala que Benjamin era filósofo, aun cuando escribiera sobre arte o literatura. Su juicio no solo presupone una clara distinción entre los objetos de saber de la filosofía y los de otros saberes. Además, asume una mayor profundidad en el primero de tales saberes. El talento de Benjamin estaría, siguiendo su diagnóstico, en haber logrado seguir siendo filósofo pese a referir a arte o literatura.

Tal diferencia y jerarquía, sin embargo, es parte de lo que el trabajo de Benjamin objeta, puesto que su preocupación por lo que tras su obra no podemos llamar tan genéricamente como "literatura", o por un ámbito tan lejano a las tradicionales preocupaciones filosóficas como el de la entonces emergente cultura de masas, difícilmente resulta un capricho que acompañe una filosofía más seria. Al contrario, en esas combinaciones reside parte de su singular trabajo crítico, erigido, de acuerdo a Adorno, frente a la concepción tradicional de la filosofía. Ante ella, Benjamin abre la posibilidad de retomar el trabajo filosófico más allá de su institucionalización disciplinar. Adorno, por ello, lo describe como guardián del tesoro filosófico (1995a, p. 73). En particular, Adorno rescata al respecto la Obra de los pasajes, trabajo que no lee al margen de la filosofía. Antes bien, para Adorno 
este tipo de obra es más filosófica que el de la institucionalidad filosófica, debido a la obsesión detallista marcada por la exigencia de pensar lo que debe ser pensado: "El reto de escribir un artículo sobre los pasajes de París contiene más filosofía que las consideraciones sobre el ser del ser" (Adorno, 1995a, p. 15).

Adorno no es el único, por cierto, quien sitúa a Benjamin en la filosofía. Podemos recordar también que Lacoue-Labarthe (1992, p. 423) indica que su crítica a la institucionalidad universitaria se erige desde la filosofía. $\mathrm{Y}$ es esto lo que exponen algunas lecturas de las últimas décadas, que no podríamos resumir acá, en torno a cuestiones filosóficas de su obra que, según Subirats (1991, p. 9), las primeras lecturas de Benjamin desconsideran. Para ello, variados lectores han explorado algunos conceptos centrales en su obra (véase, entre muchos ejemplos posibles, Weber, 2008), y otros tantos han mostrado algunas de las afinidades y tensiones con otros autores a los que sigue resultando más cómodo llamar filósofos. Así, por ejemplo, Jay (2003, p. 71) instala su reflexión a partir de los debates sobre la experiencia desarrollados por Dilthey, Husserl o Junger; Derrida (1997, p. 74) remarca su afinidad epocal en la crítica de la democracia parlamentaria de Heidegger, Schmitt o Leo Strauss; y De Man (1990, p. 145) lo emplaza en las lecturas de lo sublime acerca de la estética hegeliana. Frente a tales posicionamientos, sin embargo, también en destacados autores contemporáneos pueden hallarse lecturas que sitúan a Benjamin fuera de la filosofía. De este modo, para Jameson (1992, p. 32), Benjamin no pareciera ser un autor filosófico.

Este rápido y burdo sondeo, o cualquier otro que recordase a otros lectores de Benjamin, expresa cierta imposibilidad de determinar la escritura benjaminiana dentro o fuera de la filosofía. Y quizás la pregunta que abre esta noticia ya no es la de si Benjamin era o no un filósofo, sino qué hace su trabajo a la filosofía una vez que la hace perder su tradicional posición rectora de cualquier saber. En tal sentido, la preocupación benjaminiana por la literatura ya no podría pensarse desde un criterio filosófico que fundamente su propia seguridad. Antes bien emerge desde una reflexión filosófica que asume la incerteza del límite entre filosofía y literatura. Bien indica Gagnebin (1997, p. 80) que ese gesto cuestiona más la distinción canónica entre ambos géneros que la posición de Benjamin como filósofo. Antes que señalar, como Sarlo (2000, p. 43), que Benjamin se ubica en el espacio literario y en el filosófico porque su proyecto crítico alearía reflexiones estéticas y filosóficas, lo importante resulta entonces repensar qué puede seguir pen- 
sándose como filosofía en el siempre riesgoso espacio de pensamiento que ni se asume literario ni se asume que puede determinar dónde comienza, o termina, algo así como "lo literario"1.

Frente a cualquier sustancialización de lo que sería la filosofía, la literatura o la relación entre una y otra, con Benjamin la alternativa es la de pensar históricamente esa relación, partiendo por lo que pueda considerarse como "historia" o como "filosofía", aun cuando la alternativa de pensar una historia de la historia de la filosofía con Benjamin parece haber pasado inadvertida. Es sintomático, en esa dirección, que el texto sobre historia cultural en la Cambridge Companion sobre Benjamin (Caygill, 2004) parta refiriendo a la búsqueda benjaminiana de una nueva relación entre arte y filosofía para luego reducir tal discusión a lo que escribe Benjamin acerca de las prácticas artísticas, olvidando la chance de situar ahí a la filosofía. En lugar de inscribir su desarrollo en la historia, se le piensa como pensamiento no histórico sobre la historia. Sustraída esta de la historia cultural, se la resitúa en el sitial que Benjamin objeta: como una teoría de los objetos del pensamiento cuya historia parece estar desligada de los objetos entre los cuales se emplaza.

No está de más aquí recordar que Benjamin se forma en una universidad alemana preocupada por la relación entre historia y conocimiento que instala Dilthey. Esta pregunta por la historicidad de la cultura no solo es retomada por un neokantismo que Benjamin cuestiona por prologar la filosofía del sujeto. También destaca en autores por los que Benjamin se interesa, como Simmel o el propio Warburg. A sus perspectivas, Benjamin busca añadir una mirada destructiva que supere la archivación del pasado para hacer allí saltar la linealidad del tiempo que la retórica de la cultura supone y condensa: "la historia de la cultura representa solo aparentemente un avance de la comprensión y ni siquiera aparentemente representa un avance de la dialéctica. Porque le falta el momento destructivo que garantiza tanto la autenticidad del pensamiento dialéctico como la de la experiencia del dialéctico mismo. Desde luego que aumenta el peso de los tesoros amontonados en las espaldas de la humanidad. Pero no le da a ésta fuerzas para sacudirlos y tenerlos de este modo en las manos" (Benjamin, 1973, p. 102).

\footnotetext{
${ }^{1}$ Algo similar, por cierto, podríamos argumentar con respecto a la también huidiza relación de Benjamin ante la política. Buck-Morss (1998), por ejemplo, señala que en su obra filosofía y política se identifican. Frente a ello, parece necesario eludir una identificación tan rápida como esa. Algunos trabajos de la misma intérprete podrían ser útiles para ello.
} 
En las primeras páginas de su tesis sobre el concepto de crítica en el romanticismo alemán, Benjamin tematiza la posibilidad de pensar ciertas cuestiones a partir de cierta historia filosófica. En efecto, inscribe su investigación dentro de la historia de los problemas, indicando que se ocupará de uno. A saber, el de la crítica. Por ello, distingue su historia de los problemas de la historia de la filosofía, pues aísla el problema que ha de seguir de los aparatajes conceptuales mayores en los que se inserta (Benjamin, 2007a, pp. 13-19).

En tal dirección, este trabajo de Benjamin podría ser leído como un heterodoxo antecedente de los posteriores desarrollos de la posterior historia conceptual, ya que su investigación busca rastrear las afinidades y mutaciones del sentido de un vocablo inagotable y necesario a cada una de sus particulares inscripciones. Sin embargo, su estrategia se establece con la consideración de una verdad previa a tales presentaciones discursivas -lo cual, claro está, harto dista del proyecto de Koselleck (cfr., 1993). Y es que, para Benjamin, los problemas que una época piensa no surgen de su tiempo, sino a la inversa: la época es pensada por la intemporalidad que la atraviesa. El abordaje sistemático de las variaciones de un concepto en la historia permite entonces comprender una etapa en la que se expresan los cambios de cuestiones eternas que históricamente se modifican.

Esta singular estrategia se tematiza en el críptico prólogo que abre la siguiente investigación sistemática benjaminiana, desplegada en torno al drama barroco alemán. Como es sabido, Benjamin retoma la noción platónica de la idea para pensarla desde sus variadas expresiones. A diferencia de Platón, no piensa una idealidad previa a sus múltiples inscripciones históricas. Antes bien, la tematiza como cierta idealidad que se presenta, de modo siempre inacabado y plural, sin una presencia previa a sus manifestaciones. El pensamiento, ante esa multiplicidad, ha de pensar su identidad en su diversidad fenoménica, y notar en ese índice la transformación histórica. Un abordaje histórico, por tanto, no significa una recolección lineal de datos, sino la pregunta por cómo se inscriben y relacionan los finitos e incansables fenómenos en la historia, en tanto exposición de la búsqueda por lo que excede la historia.

Desde la pregunta por un fenómeno temporal puede así enmarcarse la indagación por intemporales ideas, asumiendo la necesidad de un salto discontinuo entre uno y otro plano, dada la imposibilidad de alcanzar la idea mediante una simple inducción. Sin ese riesgo, no se podría aspirar a 
pensar una verdad irreductible a cualquiera de sus presentaciones. La verdad, por tanto, relampaguea en donaciones que exceden cualquier saber del sujeto sobre cualquier fenómeno que remite a la idea. Y esta imposibilidad de un conocimiento certero de la idea no autoriza al investigador a la renuncia. Todo lo contrario, genera una búsqueda necesaria e imposible. Bien dice Oyarzún (2009, p. 229), por ello, que Benjamin traza un método filosófico que nace con metas que son, de antemano, irrealizables.

En ese sentido, la posterior y más conocida imagen benjaminiana del paso del cepillo a contrapelo a la historia no significa que la filosofía se oponga a la historia, como lo piensa Mosès (1997, p. 101). Antes bien, de lo que se trata es de leer de otro modo lo que la historia da a pensar, en la sustracción de la continuidad histórica de lo que no pudo sino manifestarse históricamente. Tal como puede realizarse una historia filosófica desde el drama barroco alemán hacia la idea de ese drama, también puede escribirse cierta historia filosófica de una u otra idea filosófica, en el entendido de que lo que busca la filosofía, en sus distintas manifestaciones históricas, son justamente esas ideas (Benjamin, 2007b, p. 233).

Sin embargo, una revisión de lo escrito por Benjamin en este y otros textos muestra que no es alta su consideración de lo que ha pensado lo que solemos llamar como historia de la filosofía. De hecho, suele discutir con autores ajenos al canon, $y$ valerse del canon para ejemplificar cuestiones también renuentes a una tradicional historia de la filosofía. De este modo, a propósito del drama barroco alemán, los trabajos filosóficos discutidos son los de Nietzsche o Schmitt, mientras que Descartes resulta un ejemplo de la expresión barroca, Kant de la imagen del melancólico y el romanticismo del aprendizaje de la sonoridad barroca. Sus nombres propios pierden una eventual posición rectora de la conciencia de la época, para pasar antes a ser datos de un tiempo que no habrían podido pensar con la radicalidad necesaria. Por lo mismo, este gesto de destronamiento del discurso filosófico tradicional se replica en otros trabajos de Benjamin, en los que se vale de los filósofos como ejemplos para pensar cuestiones que el canon filosófico soslaya. De esta forma, Benjamin recuerda la similitud de posiciones de Kant y Mozart en torno al matrimonio (2007c, pp. 127-129), de una carta de Hegel a su esposa a propósito de la emergencia de la multitud urbana en París (1998a, p. 137) o a Schopenhauer como ejemplo de la generación que se retira de la fotografía para dirigirse hacia su espacio privado (2007d, p. 395). 


\section{LA FILOSOFÍA EN LA HISTORIA DE LAS IDEAS}

En tal sentido, la historización de la filosofía en Benjamin solo puede pensarse desde cierta concepción más amplia de la historia que busca las ideas con o sin lo que hoy comprendemos como filosofía. Antes que dar por supuesta una forma natural de filosofía, Benjamin se pregunta por su histórica presentación y diferenciación ante otros saberes. La posibilidad de su emergencia se vincula para Benjamin a la interrupción del mito (2007c, p. 172). Frente a quien leyera allí la reiteración de un tópico racionalista, para Benjamin este paso no ha de pensarse de modo teleológico y conclusivo. Antes bien, es porque el mito siempre podría interpretar que la filosofía, en tanto búsqueda de la idea, debe insistir, una y otra vez, en su diferencia con el irracionalismo del mito en cualquiera de sus versiones. Así, es con Heráclito que Benjamin interpreta la lectura de Hölderlin sobre la unidad griega entre hombres y dioses (Benjamin, 2007a, p. 124). Antes que suponer una unidad prerreflexiva entre lo humano y lo divino, la filosofía pareciera nacer como pregunta por esa relación y cesura que retoma el poeta alemán, contra una historia de la filosofía que hace siglos la ha olvidado. Ya Platón, según Benjamin, sacrifica a los dioses el saber socrático, ofreciendo su saber a los dioses del mito (2007j, p. 134).

Frente a ello, Benjamin busca pensar contra cualquier figura de la razón como destino, defendiendo al pensamiento de cualquier mito. En particular, ante su posterior vuelco hacia el marxismo, el cual lo obliga a complejizar, a partir de un abordaje clasista, la lectura de la historia de la filosofía. La indagación por la idea deviene entonces una búsqueda política frente a lo que la mitología de la razón burguesa no puede pensar en su deseo de objetivar el mundo. Una vez que Benjamin piensa la cultura en la historia de la barbarie, la cuestión de la filosofía ya no puede ser pensada como una mera interrupción del mito, sino como una crítica a toda razón que crea estar fuera de la historia del mito. Esto es, en oposición a la filosofía moderna del sujeto y su pretensión de autofundamentación. Descartes, de hecho, es para Benjamin ejemplo de quien construye desde cero (Benjamin, 2007d, p. 218).

Tras la obra cartesiana, Benjamin asume que la filosofía comienza a delimitarse, frente a la teología o la literatura. Es Leibniz, para quien nos interesa, el último ejemplo filosófico de la imagen señorial del sabio, la que luego retoman, al margen de la filosofía, Bachofen y Warburg (Benjamin, 2007f, 
p. 228). En paralelo, tras el pensamiento cartesiano, según Benjamin la filosofía se cierra a esa apertura, refugiándose en la pretensión de certeza de quien piensa. Es en ese momento donde Benjamin emplaza a Kant (2007i, p. 163). En el resguardo de su exterior, la filosofía crítica pierde la alternativa de pensar lo que la rodea. Benjamin, en efecto, se refiere allí a cierto signo de la interioridad (1973, p. 121). La universalidad que pretende es la de una clase dominante que cree, al observarse, observar toda experiencia de la humanidad. Con ello, pierde la experiencia, y con ella la posibilidad de pensar de otro modo que desde sí misma: "Cuando se está hablando de la humanidad no debe olvidarse la estrechez del aposento burgués en el que nació la Ilustración” (Benjamin, 1995, p. 85).

En esa línea, Benjamin que supone que la aproximación filosófica de la idea se halla condicionada por los posicionamientos ante los modos de producción de saber. Con ello, por cierto, no soslaya la intemporalidad de la idea, como podría hacerlo alguna sociología del conocimiento. Por el contrario, puesto que la idea excede cualquier paradigma o campo intelectual es que puede modularse de más de una forma, lo que permite a Benjamin imaginar una filosofía que le haga justicia a su promesa, más allá de los individualizados límites de la epistemología moderna. Al encerrarse en su deseo de autofundamentación, esta última deviene incapaz de pensar los efectos históricos de una metafísica de la subjetividad que tecnifica al mundo. Ante ello, el kantismo deriva hacia una filosofía conservadora como en el tardío Schlegel (Benjamin, 2007a, p. 36) o hacia algunas abstracciones metafísicas sobre la guerra. Es decir, cierta reflexión sobre la técnica incapaz de pensarse en su posicionamiento histórico (Benjamin, 1991, p. 55). Tal como sus antecesores liberales, los filósofos del fascismo piensan en una universalidad abstracta del hombre, incluso a la hora de reflexionar sobre las transformaciones entre "el hombre" y "el mundo".

Frente a ello, Benjamin nota que otras filosofías buscan ampliar la pregunta por la experiencia más allá de la técnica, pero de modo también ahistórico. Es allí donde sitúa la obra de Bergson, comprendida como parte de una época en la que la subjetividad ha perdido la posibilidad de dirigir su juicio. En su lectura, Benjamin se desliga de una pregunta genérica por el momento histórico de los autores, al modo de una retórica del espíritu de la época, emplazando un análisis materialista que no olvida el espacio concreto en el que viven. Al naturalizar su condición, precisa Benjamin, el filósofo francés olvida que su reflexión es habilitada por una experiencia inhospitalaria en la era de la gran industria que fragmenta la conciencia (Benjamin, 1998a, p. 126). 
De este muy rápido repaso por algunas caracterizaciones benjaminianas de la historia de la filosofía se sigue la necesidad de leer, con y más allá de tales indicaciones, las marcas de la historicidad de la barbarie en las obras de los autores. Esto pide superar un análisis que se limite al pensarse histórico, por mencionar el "contexto" de un autor para tacharlo, a modo de ejemplo, de burgués. Antes bien, la tarea que abre Benjamin es la de explicar cómo es que su experiencia burguesa marca, en su elaboración conceptual, su relación con las ideas, a partir de la diferenciación histórica de distintos modos de saber. Benjamin, sin embargo, no se detiene en ello para pensar tanto la filosofía como lo que hoy consideramos como literatura. A propósito de Bergson, por ejemplo, lo contrasta con su contemporáneo Proust. Como en la de Baudelaire, en su obra destaca Benjamin huellas de las tensiones de su tiempo, trozos dispersos de una auténtica experiencia histórica a la que la filosofía habría traicionado (Benjamin, 1998a, p. 160).

\section{LAS VERDADES DE LA LITERATURA}

Es claro que la preocupación benjaminiana por tales escritores no se explica por la posibilidad de hallar en sus obras algo así como un discurso más filosófico que el de la metafísica de su época. Mucho menos por la estrategia, igualmente naturalizante de cierta forma de comprender "la filosofía" y "la literatura", de leer la textualidad literaria desde y para discusiones filosóficas a las que la literatura sirve de ejemplo. Antes bien, su interés se explica porque la literatura, desde su especificidad histórica, pareciera resultarle mucho más interesante para reconstruir alguna idea, gracias a su exposición a las tensiones que el saber filosófico habría renunciado a pensar. Es claro, por ello, que el interés de Benjamin no es el de un recurso genérico a "la" literatura, lo que replicaría las naturalizaciones que Benjamin bien critica. Antes bien, se trata del interés específico por algunos autores, que nos interesa comprender dentro de la caracterización que da Benjamin de la emergencia de la literatura moderna.

Dentro del desarrollo de la filosofía burguesa en la modernidad europea, Benjamin nota una primera separación entre la filosofía y la ciencia (Benjamin, 1998b, p. 190). La búsqueda por la idea, así, se desdobla en distintos espacios de enunciación que se redobla, tres siglos después, en la separación entre filosofía y literatura. Recién entonces pareciera posible referir a la literatura, una vez que emerge que una escritura de ficción es capaz de tematizar, en la ficción, su condición ficticia. Y, desde ese parcial espa- 
cio de búsqueda de la idea, instalar cuestiones tradicionalmente filosóficas que el pensamiento postkantiano habría olvidado. En esa línea, Benjamin describe la analogía entre las ideas de Demócrito y los románticos sobre el lenguaje (2007a, p. 59) y, más directamente, cierta reflexión filosófica en Proust (2007j, p. 329). El espesor de la literatura gana interés filosófico en la medida en que no se confunde con la filosofía, sino que rescata la búsqueda de la Idea que el neokantismo olvida. Tanto así que ni siquiera se requiere la prosa para ello. Que el teatro épico de Brecht sea dramático, argumenta Benjamin, no implica que deje de ser filosófico (Benjamin, 1975, p. 22).

Esta pérdida de prioridad de los textos del canon filosófico para la búsqueda de las ideas se profundiza una vez que Benjamin asume, con el marxismo, la crítica a cualquier pretensión de transparencia del sujeto ante sí mismo. La filosofía, a partir de tal sospecha, resulta el trabajo más avanzado de una consciencia que, por mucho que pensase su experiencia, no podría hacer transparente ante sí lo que le resulta constitutivamente opaco. Antes bien, expresa una ideología determinada por una clase cuya experiencia habría que interrumpir para pensar, entre otras cuestiones, las ideologías de las que la filosofía, en su pretensión de neutralidad, forma parte: "la reflexión, que orienta su punto de mira más hacia los intereses conscientes de los individuos que sobre el modo de comportamiento al que a menudo inconscientemente es impulsada su clase por su posición en el proceso de producción, esa reflexión lleva a una valoración excesiva del momento consciente en la formación de las ideologías" (Benjamin, 1973, p. 122).

\section{DESTRUCCIÓN Y DIALÉCTICA}

Benjamin, siguiendo lo argumentado, se vale de algunos escritores modernos para pensar contra cualquier figura de la subjetividad. Con ello, aspira a pensar las ideas sin la mediación de las categorías burguesas, y así abrirse a lo que trasciende la historia de la metafísica burguesa. Desde este prurito puede releerse, por cierto, la conocida discusión entre Benjamin y Adorno (Adorno, 1995c, p. 142²). En particular, en torno a la defensa benjaminiana de una crítica que no suponga, como en su concepción moderna, la distancia ante su objeto. En la concepción dominante de la crítica moderna Benjamin no solo nota cierta complicidad con la institución moderna del arte,

\footnotetext{
${ }^{2}$ Evidentemente, las distintas estrategias de lecturas de la literatura moderna de Benjamin y Adorno podrían también repensarse desde aquí.
} 
como bien destaca Burger (1998, p. 86), sino también una comprensión de la filosofía como ejercicio de la distancia. Frente a ello, como es sabido, Benjamin despliega una crítica de la crítica que se piensa como mediación. Ante ese modo institucional de la crítica, el recurso a la cita en tanto invocación directa resulta crucial para pensar lo que excede al sujeto e inscribe una experiencia cuya cercanía este ya no puede determinar.

Adorno lee en esa estrategia benjaminiana la sustitución de la mediación filosófica por el creciente interés político de Benjamin (cfr. Buck-Morss, 1981, p. 312). Frente a ello, Adorno sostiene que si puede haber filosofía es por la ausencia de ese inmediato instante revolucionario. Para Benjamin, por el contrario, en el instante del shock es que se abre la posibilidad del pensar gracias a la inscripción de lo que luego la lectura ha de recuperar. En esa dirección, para Benjamin la politización del instante no se erigiría contra la vocación filosófica, sino que la rescata desde su sentido más profundo.

Explicitar esta última cuestión es necesario, dado que las dificultades adornianas para comprender la articulación tardía de filosofía y marxismo en Benjamin se replican en otros autores. Por ejemplo, Arendt describe en Benjamin cierta correspondencia metafórica entre estructura y superestructura (Arendt, 1971, p. 24). Siguiendo su argumento, Benjamin nota en los fragmentos al mundo. En el fenómeno, entonces, para Arendt podría leerse la idea en su completitud sin fragmentación alguna. Para Eagleton, por su parte, el error de Benjamin es el de desvincular lo que se presenta en la historia y la verdad histórica. Por este motivo, el crítico inglés cuestiona la supuesta desconsideración benjaminiana de los pesos causales y relativos de una supuesta totalidad que necesita jerarquías explicativas. El de Benjamin, por tanto, sería un marxismo algo insuficiente, si es que no ingenuo, frente al cual intenta recordar la existencia de clases sociales: "El marxismo imagina que, para escuchar a Bach, hay que trabajar antes, o conseguir que alguien lo haga, y que los filósofos morales no pueden discutir a no ser que las prácticas con las que han crecido desde niños hayan dispuesto esta situación para ellos" (Eagleton, 2006, p. 410).

Que en la cita Eagleton se autorice como el verdadero marxista no solo parece problemático por cierta injusticia a la apertura que señeros trabajos como los de Benjamin abrieron para una crítica literaria marxista cuya chance el inglés hereda. También lo es porque lo que Benjamin busca es pensar los fenómenos culturales en una bárbara historia que los excede y posibilita, pero que pueden aspirar a suturar desde la consideración de la diferencia entre fenómeno e idea que tanto Arendt como Eagleton olvidan. $\mathrm{Al}$ pensar las tensiones del modo de producción burgués en la literatura 
burguesa, Benjamin no piensa que la literatura sea el modo de producción, sino que no hay un conocimiento único que nos permita, de modo simple, contentarnos con uno u otro saber. La de Benjamin, entonces, no es una estrategia que no pondere históricamente la literatura, sino que busca pensar, con la literatura, la historia en la que se produce. Poder pensar la literatura no implica, por tanto, creer que en la literatura se halla la experiencia por pensar, sino que puede abrir el camino a ese pensamiento, ya que la presentación de la idea, en uno u otro espacio de enunciación, ni es una mera parte de la totalidad social ni se desliga de ella. Antes bien, se inscribe en ella denunciando su falsedad gracias a la promesa de su interrupción. La tarea de la crítica es la de insistir en esa distancia, sin creer que representa la idea ni que puede desligarse de ella.

Es por esto que la tentativa benjaminiana de pensar el fenómeno sin mediación hacia una totalidad de mayor alcance no limita la verdad a su momentánea presentación. Frente a ello, dado que ninguna idea se agota en alguna de sus presentaciones, cada una de sus manifestaciones históricas obliga a seguir pensando la idea desde la cuidadosa atención a un objeto que no podría replicar la idealidad a la que remite. Bien describe Agamben tal estrategia benjaminiana como estrictamente materialista, en el sentido de que es solo con la singularidad que se manifiesta que el pensamiento podría recuperar la promesa de justicia que relampaguea sin certeza ni mediación alguna (Agamben, 2003, p. 180). Lo que se acusa como inmediatez en Benjamin, por tanto, poco tiene que ver con una irreflexiva rapidez. Por el contrario, surge asumiendo el hiato constitutivo entre la materialidad que se piensa y lo que esta permite pensar. La jerarquía no se traza entre una y otra manifestación por pensar, sino entre la plural de prácticas y lo que estas prometen al pensar.

En su presente, a Benjamin la literatura parece haberle resultado mucho más interesante para pensar al mundo moderno. Lo interesante de su estrategia es que la ausencia de jerarquía entre las producciones de la filosofía y la literatura -o entre ella y cualquier otra producción cultural- permite pensar tanto las tensiones entre filosofía y literatura dentro de su proceso histórico de diferenciación como ligarlas a un determinado momento histórico y sus conflictos, a partir de una mirada que no se rige desde algún esquema filosófico previo a la lectura. Antes bien, el carácter filosófico de su lectura emerge con su material de lectura, y no antes de su lectura. Podemos entonces concluir señalando que su trabajo siempre habría sido, desde sus propias concepciones de la filosofía, filosófico. Y también, y esto es más sugerente para pensar más allá de Benjamin y sus decisiones, que es el pro- 
pio prurito filosófico el que lo lleva más allá de los márgenes de su disciplina. Con deseo de filosofía, busca en la literatura la verdad de la literatura. Con toda la preocupación posible por la verdad, deposita su mirada con la imposible búsqueda por la idea. Y esto abre, sin esquemas que medien, la infinita invocación a leer.

\section{REFERENCIAS}

Adorno, T. W. (1995a). "Epílogo a Infancia en Berlín hacia 1900". En Sobre Walter Benjamin: recensiones, artículos, cartas (pp. 72-75). Madrid: Cátedra. (1995b). “Caracterización de Walter Benjamin”. En Adorno, T. W., Sobre Walter Benjamin: recensiones, artículos, cartas (pp. 11-27). Madrid: Cátedra. (1995c). "Carta enviada a Walter Benjamin el 18 de marzo de 1936". En Sobre Walter Benjamin: recensiones, artículos, cartas (pp. 138-146). Madrid: Cátedra.

Agamben, G. (2003). "El príncipe y la rana. El problema del método en Adorno y Benjamin". En Infancia e historia. Destrucción de la experiencia y origen de la historia (pp. 159-186). Buenos Aires: Adriana Hidalgo editora.

Arendt, H. (1971). "Walter Benjamin, 1892-1940”. En Walter Benjamin; Bertolt Brecht; Hermann Broch; Rosa Luxemburgo (pp. 7-71). Barcelona: Anagrama.

Benjamin, W. (1973). "Historia y coleccionismo: Edward Fuchs". En Discursos Interrumpidos I (pp. 89-135). Madrid: Taurus.

(1975). " ¿Qué es el teatro épico? (Primera versión). Un estudio sobre Brecht”. En Tentativas sobre Brecht (pp. 15-29). Madrid: Taurus.

(1991). “Teorías del fascismo alemán". En Para una crítica de la violencia y otros ensayos. Iluminaciones IV (pp. 47-58). Madrid: Taurus.

(1995). “Johann Henrich a Immanuel Kant". En Personajes alemanes (pp. 83-86). Barcelona: Paidós.

(1998a). "Sobre algunos temas en Baudelaire". En Poesía y capitalismo. Iluminaciones II (pp. 123-170). Madrid: Taurus.

(1998b). "París, capital del siglo XIX”. En Poesía y capitalismo. Iluminaciones II (pp. 171-190). Madrid: Taurus.

(2007a). "El concepto de crítica de arte en el romanticismo alemán”. En

Obras. Libro 1, Volumen 1 (pp. 7-121). Madrid: Abada. (2007b). "El origen del drama barroco alemán". En Obras. Libro 1, Volumen 1 (pp. 217-459). Madrid: Abada.

(2007c). "Las "Afinidades Electivas" de Goethe". En Obras. Libro 1, Volumen 1 (pp. 123-215). Madrid: Abada. (2007d). “Pequeña historia de la fotografía”. En Obras. Libro II. Volumen I (pp. 377-402). Madrid: Abada. (2007e). "Dos poemas de Friedrich Holderlin". En Obras. Libro II. Volumen I (pp. 108-130). Madrid: Abada. 
(2007f). “Sócrates”. En Obras. Libro II. Volumen I (pp. 133-136). Madrid: Abada.

(2007g). “Experiencia y pobreza”. En Obras. Libro II. Volumen I (pp. 216221). Madrid: Abada.

(2007h). “Johann Jakob Bachofen”. En Obras. Libro II. Volumen I (pp. 222-237). Madrid: Abada.

(2007i). "Sobre el programa de la filosofía venidera". En Obras. Libro II. Volumen I (pp. 162-174). Madrid: Abada.

(2007j). "Hacia una imagen de Proust". En Obras. Libro II. Volumen I (pp. 317-330). Madrid: Abada.

Buck-Morss, S. (1981). Origen de la dialéctica negativa. Theodor W. Adorno, Walter Benjamin y el Instituto de Frankfurt. México D.F.: Siglo XXI.

(1998). "Walter Benjamin: entre moda academica e Avant-garde". Conferencia brindada en la Universidad de Sao Paulo, Brasil.

Burger, P. (1998). Crítica de la estética idealista. Madrid: Visor.

Caygill, H. (2004). "Walter Benjamin's concept of cultural history". In Ferris, D. S. (Ed.), The Cambridge Companion to Walter Benjamin (pp. 73-96). Cambridge, U.K.: Cambridge University Press.

De Man, P. (1990). "Conclusiones: La tarea del traductor de Walter Benjamin". En De Man, P., La resistencia a la teoría (pp. 115-162). Madrid: Visor.

Derrida, J. (1997). “Nombre de pila de Benjamin". En Derrida, J., Fuerza de ley: el 'fundamento místico de la autoridad' (pp. 69-151). Madrid: Tecnos.

Eagleton, T. (2006). La estética como ideología. Madrid: Trotta.

Gagnebin, J. M. (1997). “Da escrita filosófica em Walter Benjamin”. En Márcio Seligman-Silva, M. (Org.), Leituras de Walter Benjamin (pp. 83-92). San Pablo: FASESP.

Jameson, F. (1992). “Benjamin's readings”. Diacritics, 22(3/4), 19-34, Commemorating Walter Benjamin.

Jay, M. (2003). “Experiencia sin sujeto: Walter Benjamin y la novela”. En Jay, M., La crisis de la experiencia en la era postsubjetiva (69-97). Santiago: Ediciones Universidad Diego Portales.

Koselleck, R. (1993). Futuro y pasado. Para una semántica de los tiempos históricos. Paidós: Barcelona.

Lacoue-Labarthe, P. (1992). “Introduction to Walter Benjamin's. 'The Concept of Art Criticism in the German Romantics"'. Studies in Romanticism, 31(4), $421-32$.

Lowy, M. (2002). Walter Benjamin: Aviso de incendio. Una lectura de las tesis "Sobre el concepto de historia". México D.F.: Fondo de Cultura Económica.

Mosès, S. (1997). El ángel de la historia. Rosenzweig, Benjamin, Scholem. Madrid: Cátedra.

Oyarzún, P. (2009). "Interrupción y silencio". En Oyarzún, P., La letra volada. Ensayos sobre literatura (pp. 222-236). Santiago: Ediciones Universidad Diego Portales. 
Sarlo, B. (2000). "El crítico literario". En Sarlo, B., Siete ensayos sobre Walter Benjamin (pp. 41-52). Buenos Aires: Fondo de Cultura Económica.

Subirats, E. (1991). “Introducción”. En Benjamin, W., Para una crítica de la violencia y otros ensayos. Iluminaciones IV (pp. 9-19). Madrid: Taurus.

Weber, S. (2008). Benjamin's - abilities. Londres: Harvard University Press.

Wilde, O. (1920). "Conferencia a los estudiantes de Arte". En Wilde, O., El renacimiento del arte inglés y otros ensayos. Trad. León Felipe. Madrid: América. 